\title{
Endogenous T cells prevent tumor immune escape following adoptive $\mathrm{T}$ cell therapy
}

\author{
Scott R. Walsh, Boris Simovic, Lan Chen, Donald Bastin, Andrew Nguyen, Kyle Stephenson, Talveer S. Mandur, \\ Jonathan L. Bramson, Brian D. Lichty, and Yonghong Wan
}

McMaster Immunology Research Centre, Department of Pathology and Molecular Medicine, McMaster University, Hamilton, Ontario, Canada.

\begin{abstract}
While the outcome of adoptive T cell therapy (ACT) is typically correlated with the functionality of the inoculated T cells, the role of the endogenous $\mathrm{T}$ cells is unknown. The success of checkpoint blockade therapy has demonstrated the potentially curative value of preexisting tumor-primed T cells in cancer treatment. Given the results from checkpoint blockade therapy, we hypothesized that endogenous T cells contribute to long-term survival following ACT. Here, we describe a therapeutic approach combining ACT with an oncolytic vaccine that allows simultaneous analysis of antitumor immunity mediated by transferred and endogenous $\mathrm{T}$ cells. We found that, in addition to promoting the expansion and tumor infiltration of the transferred T cells, oncolytic vaccines boosted tumor-primed host T cells. We determined that transferred T cells contributed to rapid destruction of large tumor masses while endogenous T cells concurrently prevented the emergence of antigen-loss variants. Moreover, while transferred T cells disappeared shortly after tumor regression, endogenous T cells secured long-term memory with a broad repertoire of antigen specificity. Our findings suggest that this combination strategy may exploit the full potential of ACT and tumor-primed host T cells to eliminate the primary tumor, prevent immune escape, and provide longterm protective memory.
\end{abstract}

\section{Introduction}

Accumulating correlative evidence suggests that host $\mathrm{T}$ cells can be activated spontaneously in response to the growing tumor through recognition of tumor antigens. Particularly, tumors harboring large numbers of mutations are more likely to activate endogenous $\mathrm{T}$ cells due to the immunogenicity of neoantigens that are recognized as foreign by the immune system, thereby providing targets for T cell attack (1). The clinical relevance of these findings has been underscored by the correlation between mutation load and outcome to treatment with immune checkpoint blockade that relies on the presence of tumor-primed $\mathrm{T}$ cell populations (2-5). However, spontaneous $\mathrm{T}$ cell responses against mutant antigens are relatively inefficient and fail to mediate tumor rejection in most cases. Indeed, the fact that only a subset of patients exhibits durable responses to immune checkpoint blockade suggests that not all patients have tumor-primed $\mathrm{T}$ cells that are sufficient to eradicate tumors once unleashed.

Adoptive $\mathrm{T}$ cell therapy (ACT) with antigen-specific $\mathrm{T}$ cells represents an excellent alternative to checkpoint inhibitor therapies for treating malignancies (6). Healthy tumor-specific T cells can be grown in vitro and infused in large numbers into patients with advanced disease, thereby overcoming insufficiency of spon-

Conflict of interest: SRW, BS, LC, and YW are inventors on patent application no. CA2017/050772 (publication number WO/2017/219150), submitted by McMaster University, which covers the use of adoptive T cell therapy and oncolytic virus vaccination combination therapy.

Copyright: () 2019, American Society for Clinical Investigation. Submitted: November 15, 2018; Accepted: September 3, 2019; Published: November 4, 2019

Reference information: J Clin Invest. 2019;129(12):5400-5410.

https://doi.org/10.1172/JCl126199. taneous $\mathrm{T}$ cell responses in cancer patients. Importantly, clinical methodologies have been developed to propagate functional tumor-specific T cells in vitro (7-9), and recent clinical successes in leukemia, melanoma, neuroblastoma, and EBV-associated malignancies have proven that ACT is a viable and efficacious strategy in humans (10-14). However, a requisite step for the success of ACT is to lymphodeplete the host prior to infusing the $\mathrm{T}$ cells, a process called preconditioning, which is highly toxic to certain patients (1517). Furthermore, although preconditioning may create a favorable environment for adoptively transferred $\mathrm{T}$ cells (i.e., the elimination of regulatory T cells and homeostatic cytokine "sinks"), it also removes preexisting tumor-primed $\mathrm{T}$ cells, making it difficult, if not impossible, to determine the role and benefit of endogenous antitumor immunity in the context of ACT.

We have previously reported that oncolytic viruses (OVs) engineered to express tumor-associated antigens can effectively engage and expand tumor-specific memory $\mathrm{T}$ cells while retaining their inherent ability to directly infect and debulk the tumor and reverse the immunosuppressive tumor microenvironment $(18,19)$. In this study, we investigated the use of rhabdovirus- and vaccinia virusbased (VacV-based) oncolytic vaccines to drive systemic expansion and tumor infiltration of adoptively transferred $\mathrm{T}$ cells, thereby enhancing the therapeutic efficacy of ACT. This rational combination resulted in complete and durable tumor regression in the absence of preconditioning, a likely result of proinflammatory cytokines induced by the virus providing support for antitumor T cell responses (20). Using this model, we simultaneously monitored the contribution of the transferred and endogenous T cells to the therapeutic effect. Our data indicate that preexisting, tumor-primed host $\mathrm{T}$ cells are critical for preventing and/or eliminating antigen escape variants for achieving a durable regression by ACT and for long-term immune memory. 


\section{Results}

Combination of ACT with oncolytic vaccines induces complete tumor regression and long-term protective immunity. Motivated by the unique ability of rhabdoviral OV vaccines (OVVs) to simultaneously trigger effective expansion of preexisting central memory $\mathrm{T}$ cells $(\mathrm{Tcm})$ in the periphery and rapid recruitment into the tumor $(18,19,21)$, we explored the combination of ACT using in vitro-differentiated tumor antigen-specific Tcm with oncolytic vesicular stomatitis virus (VSV) vaccines to treat established solid tumors. WT BALB/c mice were intradermally (i.d.) challenged with CMS5 cells, a methylcholanthrene-induced fibrosarcoma expressing a defined neoepitope derived from a mutation in the ERK2 gene (ErkM $_{136-144}$, QYIHSANVL) (22). ErkM ${ }_{136-144}$-specific CD8 ${ }^{+} \mathrm{T}$ cells from DUC18 transgenic mice (23) were cultured and expanded in the presence of IL-15, IL-21, and rapamycin, which drove acquisition of a typical Tcm phenotype $\left(\mathrm{CD} 62 \mathrm{~L}^{+} \mathrm{CD} 44^{+}\right)$(Supplemental Figure 1A; supplemental material available online with this article; https://doi.org/10.1172/JCI126199DS1). After 6 days of tumor growth, mice were treated with i.v. injection of $10^{6}$ DUC18 Tcm, $10^{8}$ PFU of VSV-ErkM, or a combination of DUC18 Tcm followed 24 hours later by VSV-ErkM. We chose to test i.v. administration of VSV exclusively, as we have previously demonstrated that this is an optimal route to achieving both antigen presentation in the periphery to boost Tcm and infection of the tumor for oncolysis and $\mathrm{T}$ cell recruitment by a VSV vaccine $(18,19)$. Indeed, intratumoral injection of VSV failed to consistently boost transferred $\mathrm{T}$ cells to an effective level and cause tumor regression (data not shown). Figure $1 \mathrm{~A}$ shows that neither Tcm alone nor VSV vaccine alone had a significant impact on tumor growth, whereas combination of Tcm and VSV-boosting vaccine induced complete tumor regression and significantly prolonged survival (Figure 1B). However, effector DUC18 T cells (Teff) (differentiated in the presence of IL-2; Supplemental Figure 1A), either alone or in combination with VSV-ErkM, failed to induce a complete and sustained tumor regression in all treated mice (Figure 1C), an outcome that was correlated with poor persistence of the transferred cells (data not shown). Although VSV vaccination was able to significantly prolong survival after Teff transfer, regression induced by Tcm plus VSV-ErkM was more consistent, and subsequent survival was significantly prolonged over Teff-based treatments (Figure 1D), consistent with our previous work and current understanding $(19,24)$.

To further determine whether Tcm plus OV (without a defined tumor antigen) alone or conventional vaccine (without oncolytic or tumor-targeting activity) alone is sufficient to achieve efficacy similar to that of Tcm plus oncolytic vaccine, we tested additional groups, including VSV-MT (lacking the ErkM transgene), a replication-deficient adenoviral vector expressing ErkM (Ad-ErkM), and the ErkM peptide adjuvanted with poly I:C/CD40 antibody (25). As shown in Figure 1, E and F, Tcm plus VSV-MT failed to control tumor growth or prolong survival, confirming that antigen-specific OV vaccination is required to expand transferred $\mathrm{T}$ cells and recruit them into the tumor. Consistent with this notion, boosting with nononcolytic vaccines was less effective than VSV vaccine, with which only a small fraction of treated mice exhibited complete tumor regression and prolonged survival (Figure 1, E and F).

To directly visualize tumor infiltration of $\mathrm{CD} 8^{+} \mathrm{T}$ cells and their localization in the tumor, we stained tumor tissues on day 5 after ACT or ACT plus vaccination. As shown in Figure 2, peritumoral distribution of $\mathrm{CD}^{+} \mathrm{T}$ cells was evident after Tcm transfer alone and Tcm plus vaccination, but a significantly larger number of $\mathrm{CD}^{+} \mathrm{T}$ cells (Supplemental Figure 2) that penetrated deep into the tumor tissue was observed following VSV boosting, confirming that OVVs offer distinct advantages over conventional vaccines in the context of ACT. Finally, 100\% of long-term survivors (60+ days) following Tcm plus VSV-ErkM treatment rejected a rechallenge with CMS5 cells 2 months after cessation of therapy and showed significantly prolonged survival, suggesting formation of effective immunological memory (Figure 1G).

Expansion and persistence of endogenous ErkM-reactive $C D 8^{+}$ $T$ cells is determined by the tumor during combination therapy. To further understand how VSV vaccine influences the fate of transferred $\mathrm{T}$ cells, we also monitored $\mathrm{T}$ cell responses in the periphery. ErkM $_{136-144}$-specific CD8 ${ }^{+} \mathrm{T}$ cell expansion in the circulation could be detected as early as 2 days after VSV vaccination, which peaked at day 5 and declined thereafter (Figure 3A), coinciding with the kinetics of CMS5 tumor regression (Supplemental Figure 1B). Although antigen-specific $\mathrm{T}$ cell responses declined after the peak, they remained at approximately $10 \%$ of circulating CD8 ${ }^{+} \mathrm{T}$ cells for more than 2 months (Figure 3A). Interestingly, further analysis using congenic markers indicated that expansion of ErkM $_{136-144}{ }^{-}$ specific T cells was dominated by adoptively transferred DUC18 cells (Thy1.1 $1^{+}$) at 5 days post treatment (dpt), but was replaced by endogenous CD8 ${ }^{+} \mathrm{T}$ cells (Thy1.2 $2^{+}$) from $12 \mathrm{dpt}$ onward (Figure $3 \mathrm{~B}$ ). To determine whether the loss of the transferred DUC18 cells from the circulation resulted from differential localization, we analyzed blood, spleen, and bone marrow on day 60 after treatment. The majority of ErkM $_{136-144}$-specific T cells were endogenous CD8 ${ }^{+} \mathrm{T}$ cells in all 3 compartments, confirming that transferred DUC18 cells indeed disappeared following tumor regression (Figure 3C). This observation prompted us to evaluate ErkM $_{136-144}$-specific $\mathrm{T}$ cell responses driven by VSV-ErkM in tumor-free (TF) naive animals compared with tumor-bearing (TB) mice. WT BALB/c mice received $10^{6}$ DUC18 Tcm 1 day prior to vaccination with $10^{8} \mathrm{PFU}$ of VSV-ErkM, and ErkM-specific T cell responses were monitored on days 5,12, and 19 after VSV treatment. Figure 3D shows that the kinetics of $\mathrm{T}$ cell expansion in TF mice were similar to those in TB animals, though the rate of contraction was significantly slower at $12 \mathrm{dpt}$. More importantly, a significantly higher percentage of detected ErkM $_{136-144}$-specific T cells were DUC18 origin (Thy1.1 $1^{+}$) at all time points in TF mice compared with TB mice (Figure 3E), suggesting that (a) DUC18 have no cell-intrinsic defects in survival and (b) their deaths are likely the result of interactions with tumor cells. This latter speculation was supported by the fact that more than $90 \%$ of $\mathrm{CD}^{+} \mathrm{T}$ cells in the tumor were Thy $1.1^{+}$on day 4 after VSV boosting (Figure 3F), but subsequently underwent a progressive apoptosis (from $32 \%$ on day 4 to $95 \%$ on day 6) coinciding with the time of tumor regression. In contrast, the expansion of ErkM-specific endogenous T cells did not peak until $12 \mathrm{dpt}$ (Figure 3B), suggesting that substantial numbers of endogenous $\mathrm{T}$ cells did not directly engage interactions with the tumor and thus persisted in the memory pool.

Combination therapy induces antigen loss in the absence of endogenous lymphocytes. The observation of effective expansion and long-term persistence of endogenous ErkM-reactive $\mathrm{CD}^{+} \mathrm{T}$ 
A
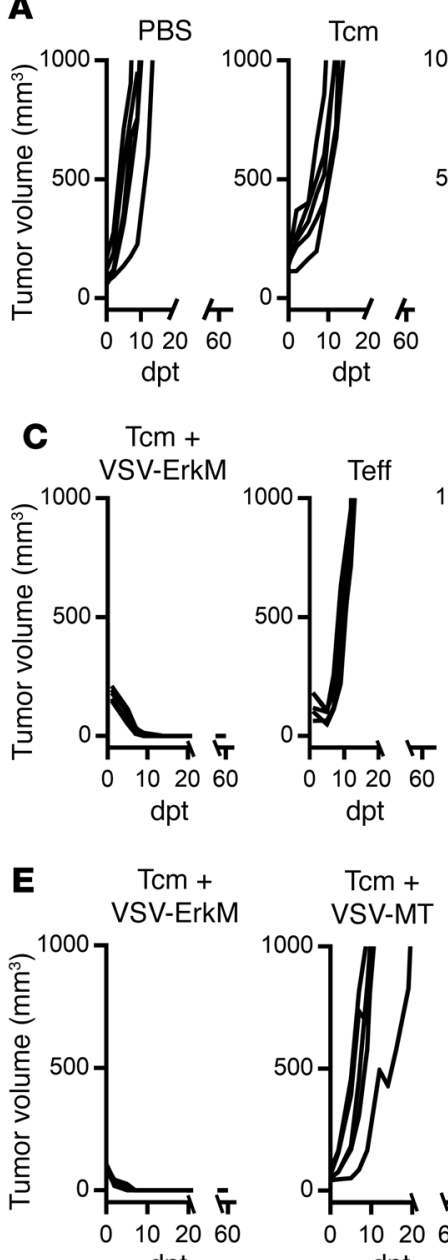

dpt
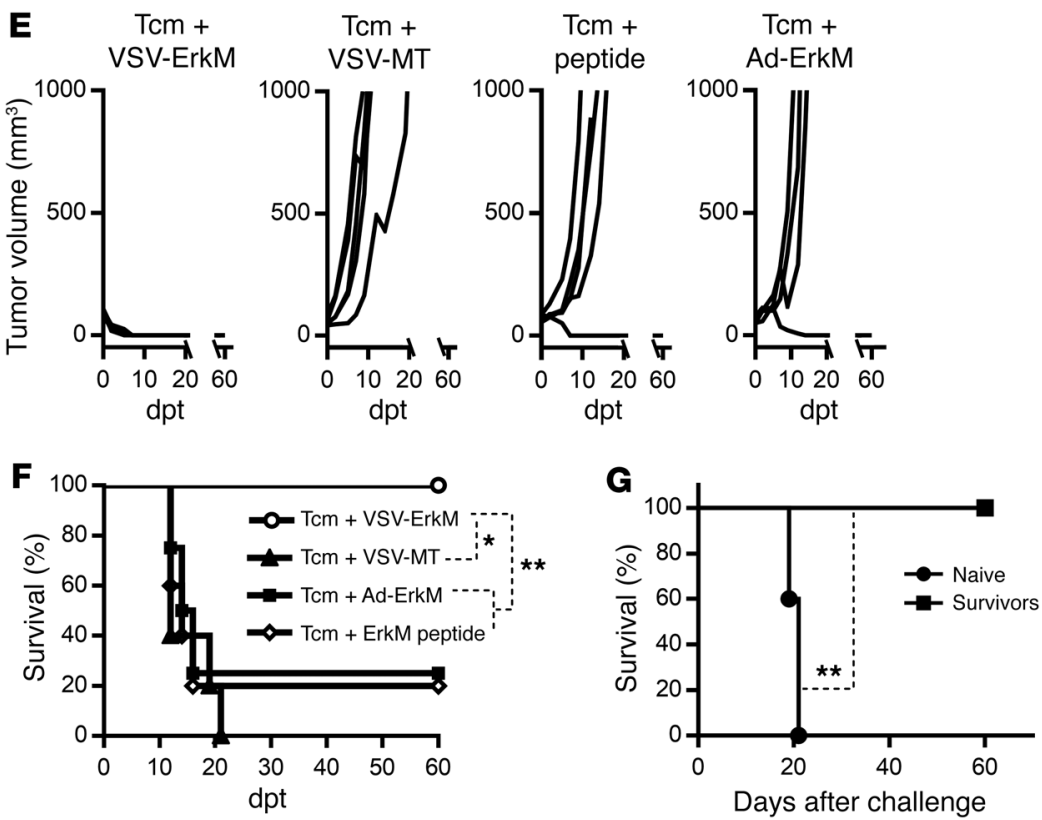

B

D
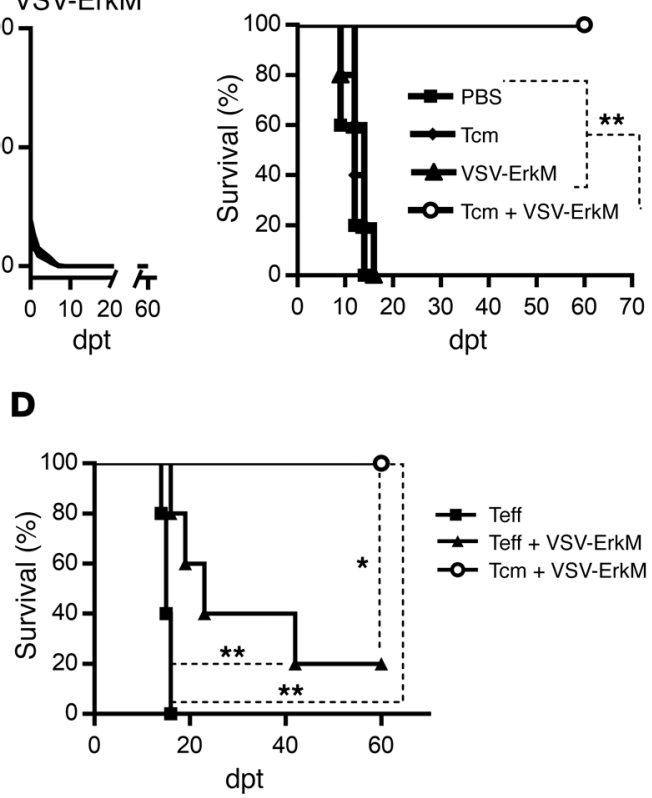

Figure 1. Combination of Tcm and VSV-ErkM leads to durable tumor regression. BALB/c mice were inoculated i.d. with CMS5 cells 7 days prior to adoptive transfer of DUC18 Tcm ( $10^{6}$ cells/mouse) and, where indicated, were treated with the specified vaccine/virus 24 hours later. Mice receiving VSV-ErkM alone, Tcm alone, or PBS were included as controls. (A, C, and E) Tumor volumes and (B, D, and $\mathbf{F}$ ) survival of CMS5 TB BALB/c mice were monitored at the specified dpt with $0 \mathrm{dpt}$ representing the day of vaccine inoculation and are expressed as $\mathrm{mm}^{3}$. A tumor volume of $1000 \mathrm{~mm}^{3}$ was used as end point for survival analysis. (G) Mice surviving initial CMS5 tumor challenge after treatment with Tcm plus VSV-ErkM were rechallenged with CMS5 cells 60 days later,and subsequent survival is shown. A group of naive mice challenged with CMS5 cells was included as a control. Data are shown as representative results from 4 (A and $\mathbf{B}), 3$ (C-F), or 2 (F) independent experiments with $n=5$ per group. Data were analyzed using a log-rank (Mantel-Cox) test (B, D, F and G). ${ }^{*} P<0.05$; ${ }^{* *} P<0.01$. cells in CMS5 TB animals prompted us to evaluate the role of host lymphocytes in combination therapy-induced tumor regression. NRG mice, which lack mature lymphocytes, were i.d. inoculated with $10^{6}$ CMS5 cells 6 days prior to combination therapy with $10^{6}$ DUC18 Tcm plus $10^{8}$ PFU of VSV-ErkM. Figure 4, A and B, shows that complete tumor regression was achieved in NRG mice, and although survival was significantly prolonged in the Tcm plus VSVErkM-treated group, the tumors in all mice eventually relapsed. Similar to the observation in WT mice, transferred T cells became undetectable in the circulation following tumor regression, but a significantly higher number of Thy $1.1^{+}$cells persisted in TF NRG mice (Figure $4 \mathrm{C}$ ), confirming that the fate of transferred $\mathrm{T}$ cells is influenced by their interactions with the tumor. We speculated that the relapsed CMS5 tumor cells might no longer express the
ErkM epitope and thus escaped DUC18-mediated elimination. To verify this possibility, we performed 2 more experiments. First, we established tumors in WT BALB/c mice using either parental CMS5 or relapsed CMS5 cells recovered from combination-treated NRG mice (CMS5r), followed by treatment with DUC18 Tcm plus VSV-ErkM. Figure 4D shows that durable regression was recapitulated in mice bearing parental CMS5 cells, but the same treatment failed to eradicate CMS5r cells, resulting in significantly reduced survival (Figure 4E), confirming that CMS5r cells cannot be recognized by ErkM-specific CD8 ${ }^{+} \mathrm{T}$ cells. Second, PCR analysis of genomic DNA from CMS5, CMS5r, and CT26 (an irrelevant control cell line) indicated that the relapsed CMS5 cells had indeed undergone a loss of heterozygosity at the ERK2 gene, resulting in elimination of the mutant ERK2 allele and therefore loss of 


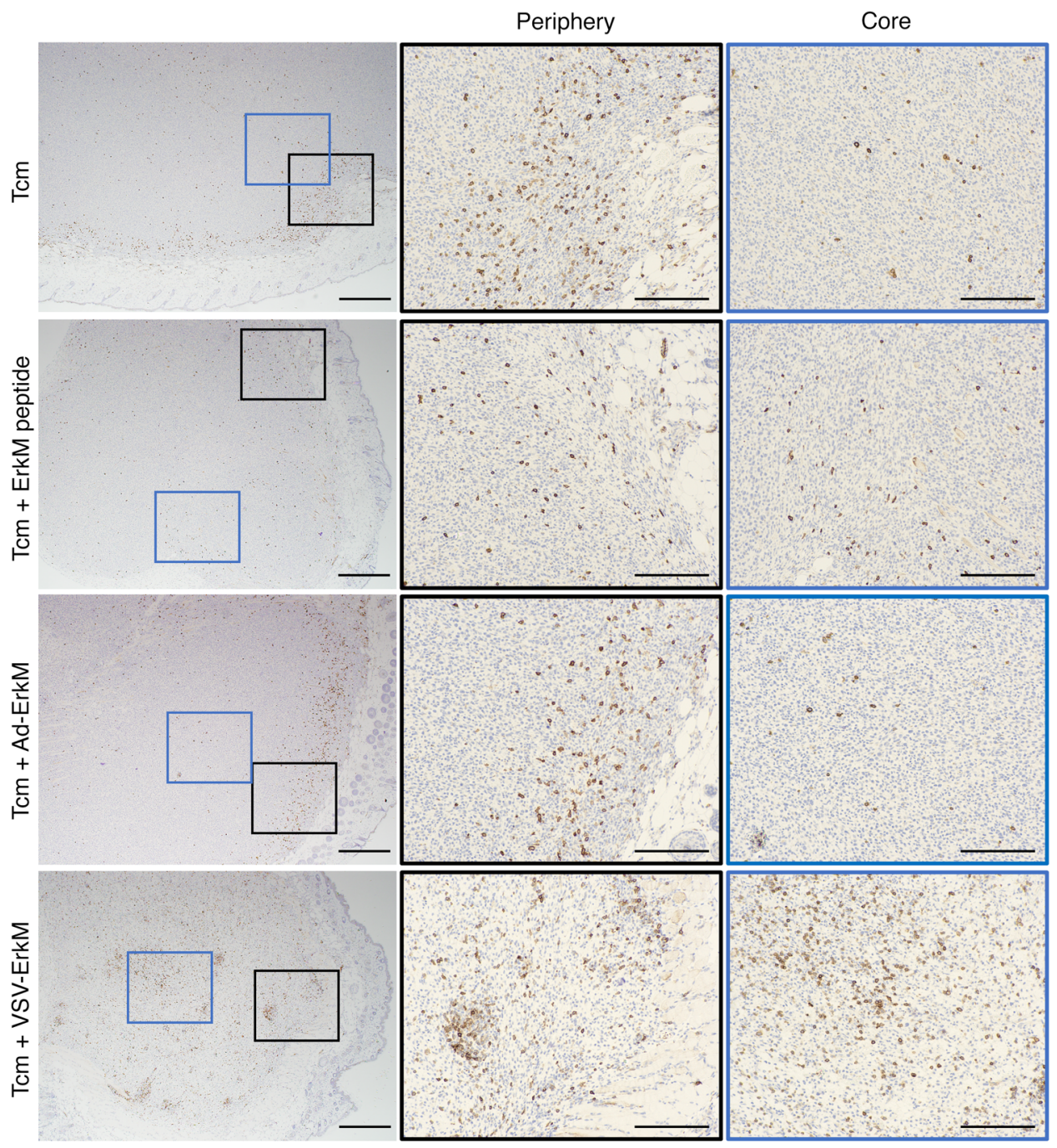

Figure 2. OVV treatment drives tumor core infiltration of transferred $\mathrm{CD8}^{+} \mathrm{T}$ cells. Micrographs of CMS5 tumor tissues stained with an anti-CD8 antibody show relative infiltration of the tumor core and periphery with $T$ cells induced by the indicated treatments. Low-magnification images of the whole tumor are shown in left panels, and higher magnification images of the tumor periphery (outlined by black boxes) and tumor core (outlined by blue boxes) are shown in the center and right panels. Scale bars: $500 \mu \mathrm{m}$ (left panels); 200 $\mu \mathrm{m}$ (center and right panels).

ErkM antigen (Figure 4, F-H). Finally, as expected, CMS5r cells were resistant to killing by DUC18 Teff cells in vitro while engineered expression of the ErkM peptide in these cells made them susceptible to killing, similarly to CMS5 cells, confirming that the resistance of CMS5r to ErkM-specific T cell lysis was due to the absence of target antigen expression, but not other alterations (Supplemental Figure 3). These results highlight an important role of endogenous lymphocytes in avoiding immune selection of antigen loss variants during ACT.

Both endogenous $C D 4^{+}$and $C D 8^{+} T$ cells are required to prevent tumor escape during combination therapy. To determine the subset of endogenous lymphocytes that are required in preventing relapse during combination therapy, we performed in vivo antibody depletion experiments in TB mice treated with DUC18 Tcm plus VSV-ErkM. Antibodies against CD4 or Thy1.2 were given 1 day before tumor inoculation, while anti-CD8 antibody was administered 1 week earlier to avoid its effect on transferred DUC18 T cells. All treated mice had tumor regression irrespective of selective depletion of endogenous lymphocytes (Figure 5A), which is similar to observations in treated NRG mice (Figure 4A) that are genetically deficient for endogenous lymphocytes. However, relapse occurred in $80 \%$ of mice that received CD8 antibody and $100 \%$ in the case of CD 4 or Thy1.2 depletion (Figure 5A), confirming that both endogenous $\mathrm{CD}^{+}$and $\mathrm{CD} 8^{+} \mathrm{T}$ cells are required to prevent tumor recurrence following treatment with ACT plus oncolytic vaccination. We also included a group that received cyclophosphamide (CPX), a commonly used chemo drug for lymphodepletion $(26,27), 1$ day before combination therapy. Similarly to what occurred with antibody treatment, CPX did not affect initial tumor regression, but all animals relapsed within 2 weeks (Figure 5A). All antibody depletion and CPX treatments resulted in a significant decrease in survival (Figure 5B)

One possible mechanism for the requirement of endogenous $\mathrm{T}$ lymphocytes is due to epitope spreading, a consequence of tumor destruction by combination therapy, which results in a second wave of antitumor attack against different antigens. This notion appeared to be supported by the fact that initial tumor regression following combination therapy did not require any endogenous lymphocytes (Figures $4 \mathrm{~A}$ and $5 \mathrm{~A}$ ). We thus hypothesized that in vivo depletion of $\mathrm{CD}^{+}$and/or $\mathrm{CD}^{+} \mathrm{T}$ cells on day 6 after ACT plus oncolytic vaccination (a time point at which tumors were still regressing) would result in recurrence. Surprisingly, however, depletion of either or both subsets did not lead to tumor relapse (data not shown), suggesting that tumor-reactive endogenous $\mathrm{T}$ 
A

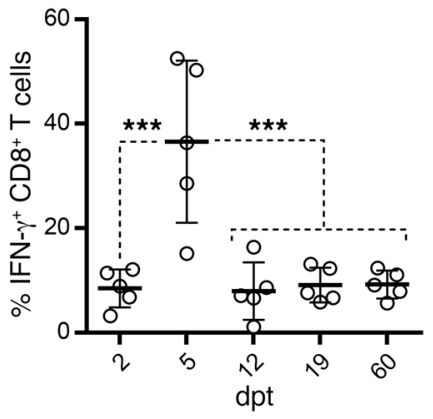

C

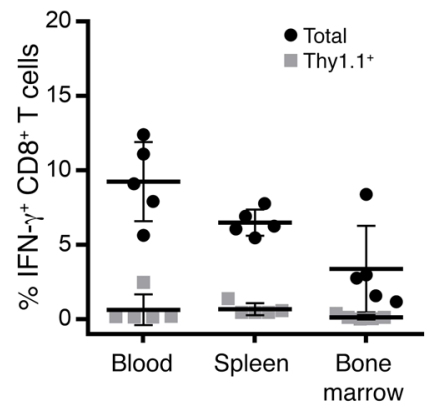

E

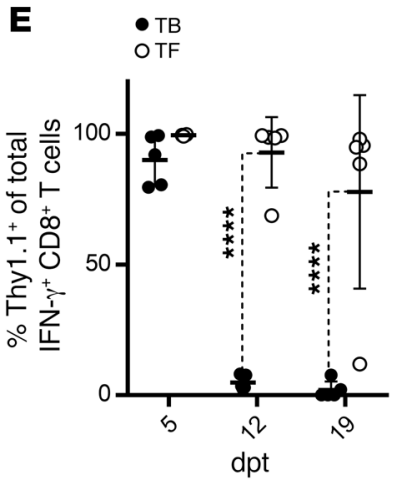

B

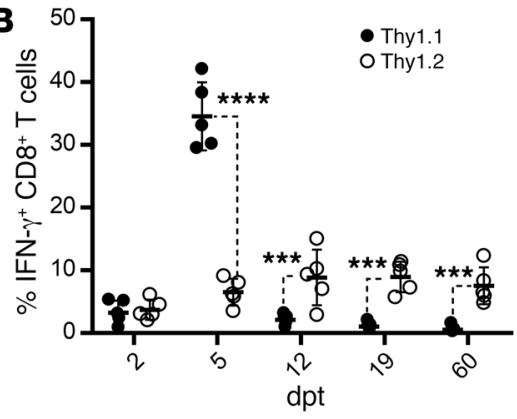

D

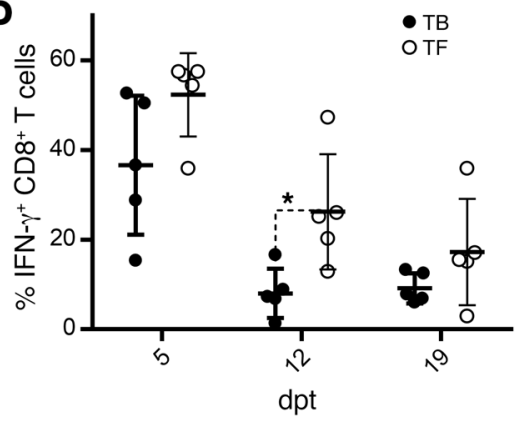

$\mathbf{F}$

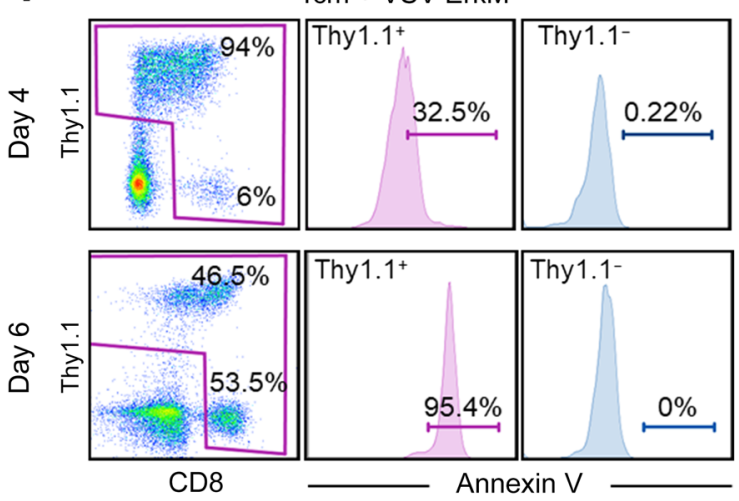

Figure 3. Expansion and persistence of ErkM-reactive $\mathrm{CDB}^{+} \mathrm{T}$ cells are influenced by tumor during combination therapy. (A) Venous blood was collected on the designated dpt. Virus component of combination therapy and frequency of ErkM-specific CD8 ${ }^{+} T$ cell responses were evaluated. (B) Proportion of adoptively transferred versus endogenous cells within the total pool of ErkM-specific CD8 ${ }^{+} \mathrm{T}$ cells in circulation was determined by antibodies specific for Thy1.1 (transferred) and Thy1.2 (endogenous) in gated IFN- $\gamma^{+}$cells at the time points indicated. (C) $\mathrm{CD} 8^{+} \mathrm{T}$ cells collected from blood, spleen, and bone marrow of mice 60 days after CMS5 tumor regression induced by combination therapy were assessed for ErkM specificity. Black dots represent total frequency of ErkM-specific CD8 ${ }^{+} \mathrm{T}$ cells, and superimposed gray boxes represent frequency of transferred ErkM-specific CD8 ${ }^{+} T$ cells. (D) Magnitude of ErkM-specific CD8 ${ }^{+} \mathrm{T}$ cell responses and (E) frequency of Thy1.1+ $T$ cells in the total pool of ErkM-specific CD8 ${ }^{+} \mathrm{T}$ cells in the circulation of TB and TF mice as assessed at time points indicated. (F) Representative flow cytometry profiles of tumor-infiltrating $\mathrm{CD}^{+} \mathrm{T}$ cells costained for Thy 1.1 and annexin $\mathrm{V}$ on days 4 and 6 after vaccination are shown. Data are representative of results of 3 ( $\mathbf{A}$ and B) or $2(\mathbf{C}-\mathbf{F})$ independent experiments with $n=5$ per group. Data were analyzed using 1-way ANOVA (A), 2-tailed $t$ test (C), or repeated measures 2-way ANOVA with Holm-Šidák correction for multiple comparisons (B, D, and E). ${ }^{*} P<0.05 ;{ }^{* *} P<0.001 ;{ }^{* * *} P<0.0001$. cells must be present before or be extremely rapidly induced by combination therapy, either of which is critical for complete eradication of tumor cells in cooperation with adoptively transferred $\mathrm{T}$ cells. Evidence that endogenous ErkM-specific CD8 ${ }^{+} \mathrm{T}$ cell expansion was observed as early as 2 days after VSV boosting (Figure 3A) favors the possibility of preexisting tumor-primed endogenous $\mathrm{T}$ cells that can be unleashed or even boosted by combination therapy to participate in early tumor clearance.

We made an attempt to determine whether $\mathrm{CD} 8^{+} \mathrm{T}$ cell responses against nontargeted antigens could be detected in the circulation. Mononuclear cells were collected from blood 5 days after ACT with or without vaccine boosting. IFN- $\gamma$ production by $\mathrm{CD}^{+} \mathrm{T}$ cells was quantified 4 hours after stimulation with a peptide pool consisting of 4 immunogenic neoepitopes identified by Duan et al. (28). As shown in Figure 5C, although the level of CD8 $8^{+} \mathrm{T}$ cell responses against pooled neoepitope peptides was negligible in mice that received ACT only, it could be significantly boosted by vaccination with an oncolytic vaccine. This result, together with data shown in Figure 2A, confirmed that tumor-primed endogenous T cells specific to both targeted and nontargeted antigens were present and could be rapidly amplified by oncolytic vaccination.
Endogenous $T$ cells form long-term antitumor immunity in animals following combination therapy. To determine the persistence and protective function of endogenous $\mathrm{T}$ cells, especially those that recognize nontargeted tumor antigens, we decided to rechallenge survivors ( $\mathrm{TF}$ for $>60$ days following elimination of initial CMS5 tumors) with an i.d. injection of CMS5r cells. We also included a group rechallenged with parental CMS5 cells as an analytical control. Five days after rechallenge, we stimulated sampled PBMCs with the ErkM peptide and compared the frequency of IFN $-\gamma^{+} \mathrm{CD}^{+} \mathrm{T}$ cells to that in the same animals before rechallenge. As expected, an approximately 3-fold increase of ErkMreactive $\mathrm{CD}^{+} \mathrm{T}$ cells was observed in survivors rechallenged with CMS5, while rechallenge with CMS5r did not boost any ErkMspecific $\mathrm{CD}^{+} \mathrm{T}$ cell responses, reinforcing the fact that CMS5r lost ErkM expression (Figure 5D). Interestingly, however, neither cell lines could form tumors in survivors, though both grew rapidly in untreated mice and significantly reduced survival (Figure $5 \mathrm{E}$ ), suggesting that memory against ErkM as well as other antigens persisted as a consequence of successful combination therapy and was sufficient to mediate protection against a rechallenge with either parental or ErkM-negative CMS5 cells. Furthermore, 

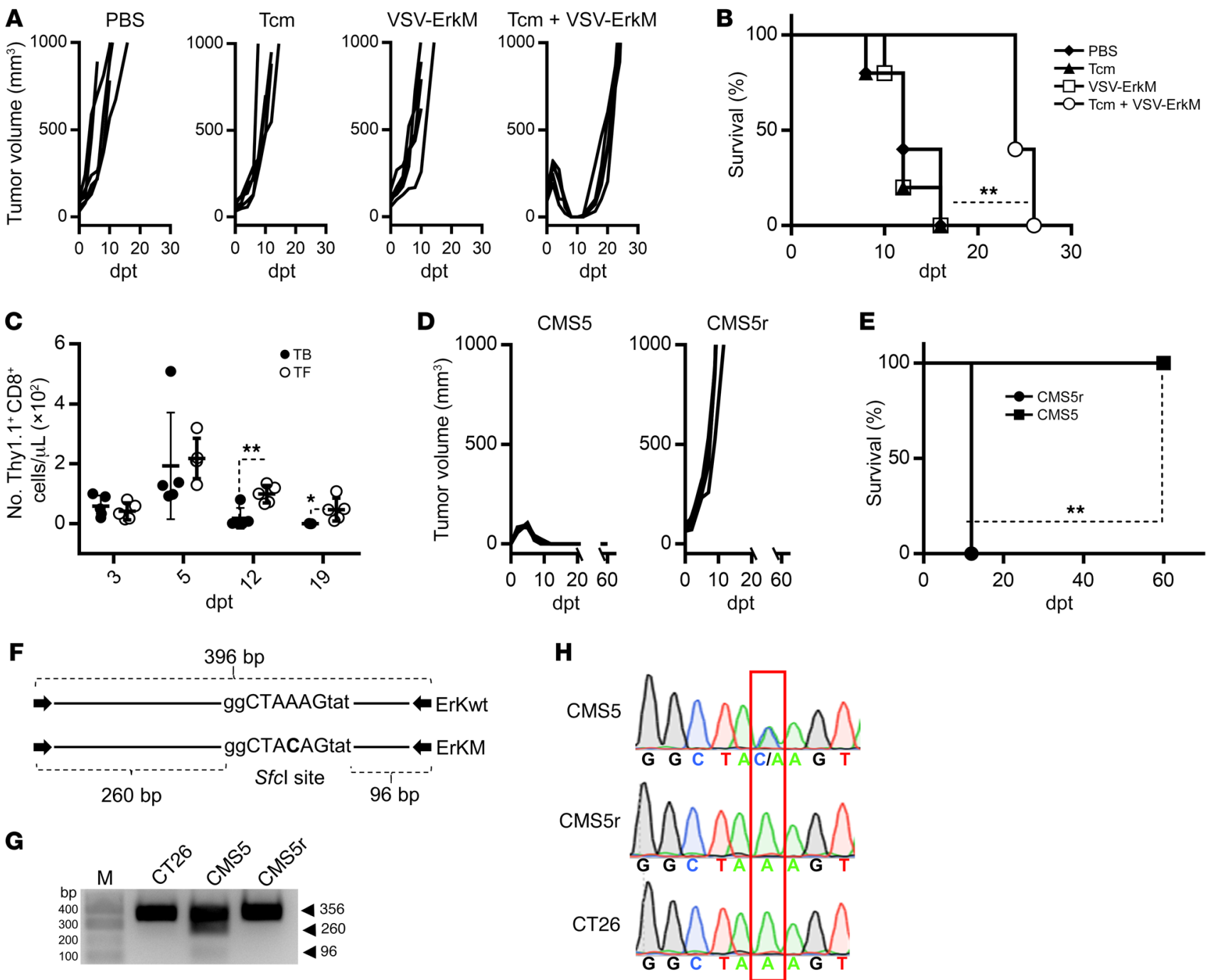

Figure 4. Endogenous lymphocytes prevent outgrowth of antigen-negative tumor cells. NRG mice were inoculated i.d. with CMS5 cells 7 days prior to adoptive transfer of DUC18 Tcm ( $10^{6}$ cells/mouse). One day after Tcm transfer, mice were vaccinated i.v. with VSV-ErkM ( $\left.2 \times 10^{8} \mathrm{PFU} / \mathrm{mouse}\right)$. Mice receiving VSV-ErkM alone, Tcm alone, or PBS were included as controls. (A) Tumor volume and (B) survival of treated mice is shown at the indicated dpt. (C) The numbers of transferred (Thy1.1+) CD8 ${ }^{+}$T cells in the peripheral blood of TB and TF NRG mice on days 3, 5, 12, and 19 after combination therapy were determined by flow analysis. WT mice surviving initial CMS5 tumor challenge after treatment with Tcm plus VSV-ErkM were rechallenged with CMS5 relapse (CMS5r) cells 60 days later, and subsequent tumor growth (D) and survival (E) are shown. Naive mice that received CMS5 were included as controls. Data are shown as representative results of $3(\mathbf{A}$ and $\mathbf{B})$ or $2(\mathbf{C}-\mathbf{E})$ independent experiments with $n=5$ per group. Data were analyzed using repeated measures 2-way ANOVA with Holm-Šidák correction for multiple comparisons (C) and log-rank (Mantel-Cox) test (B and $\mathbf{E}) .{ }^{*} P<0.05$; ${ }^{* *} P<0.01$. (F) Schematic of the PCR product resulting from genomic DNA amplification of the ERK2 gene is shown with the $S f c l$ recognition sequence generated by the ErkM mutation displayed in uppercase letters. Expected fragments generated from Sfcl digestion of PCR amplicons from WT ERK (356bp bands for ErKwt) and mutant ERK alleles (260 and 96 bp bands for ErKM) are shown with dotted brackets. (G) Restriction digestion of PCR products amplified from CT26 (negative control), CMS5, and CMS5r cell line genomic DNA is shown as well as $(\mathbf{H})$ chromatogram of sequencing result from PCR products.

as additional evidence of shared antigens in exception to ErkM, mice vaccinated with lethally irradiated CMS5r cells (irrCMS5r) were protected from subsequent challenge with either CMS5 or CMS5r cells (Figure 5F).

To determine which lymphocyte population was required for protection against CMS5r, we carried out antibody-mediated depletion of $\mathrm{CD}^{+}, \mathrm{CD}^{+}$, or both $\mathrm{T}$ cell subsets in combination therapy survivors before rechallenge. $\mathrm{CD}^{+} \mathrm{T}$ cell depletion resulted in tumor growth in $80 \%$ of challenged animals, while all mice that received antibodies against CD 4 or both CD 4 and CD8 T cells developed tumors, causing significantly reduced survival in all depletion groups (Figure 5G). These results demonstrate that both subsets of endogenous $\mathrm{T}$ cells are not only critical for preventing the emergence of or eliminating preexisting antigen-loss variants during ACT, but also for forming long-term protective immunity with broad antigen specificity.

Requirement of endogenous $T$ lymphocytes for preventing tumor relapse is not antigen and/or model dependent. Finally, we sought to evaluate the combination platform of ACT plus OV vaccination in a different tumor model to determine the role of endogenous $\mathrm{T}$ lymphocytes in achieving complete and durable tumor regression. To this end, we chose to use $\mathrm{VacV}$ as an oncolytic viral 
A
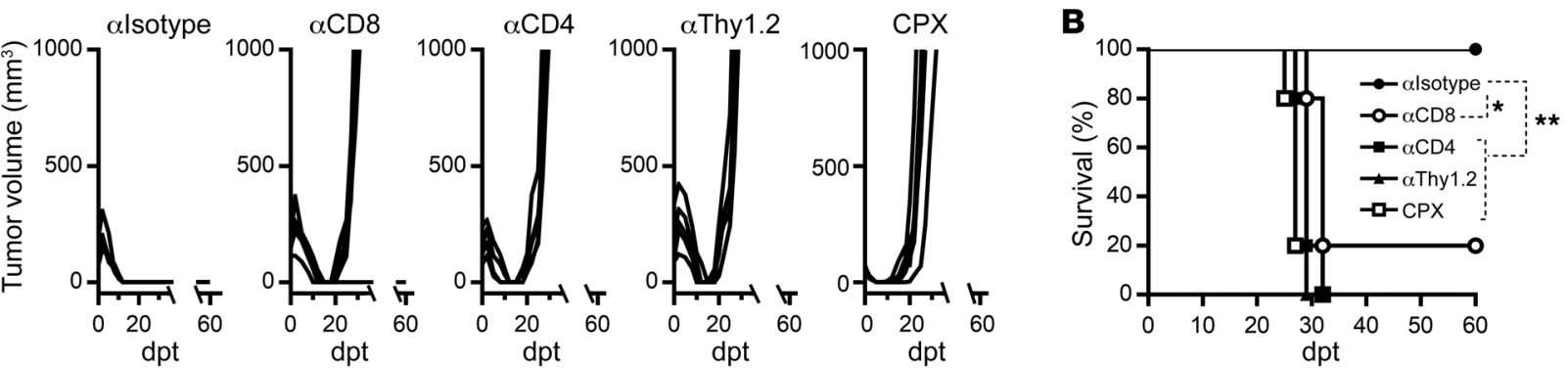

C

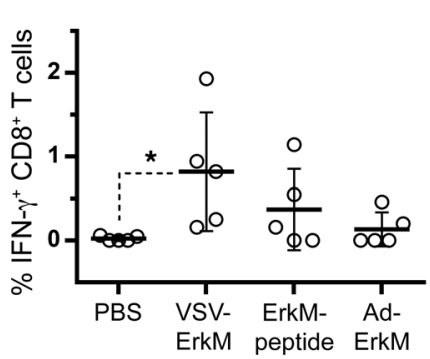

$\mathbf{F}$

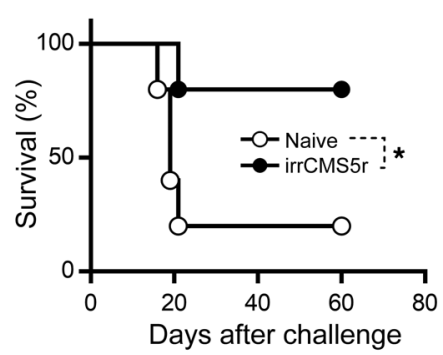

D

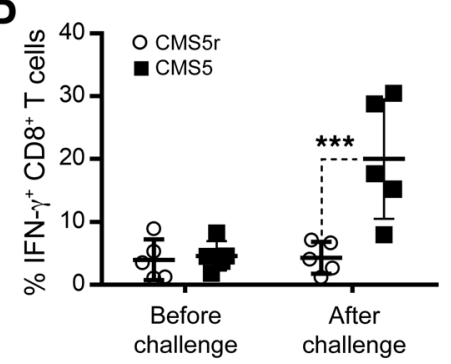

E

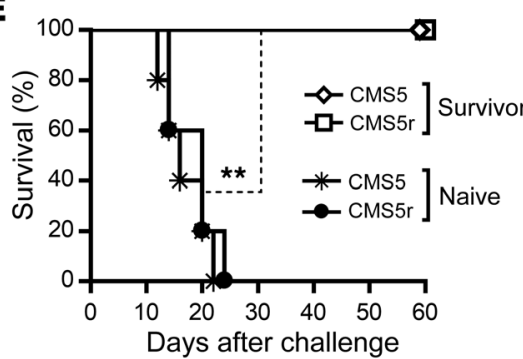

G

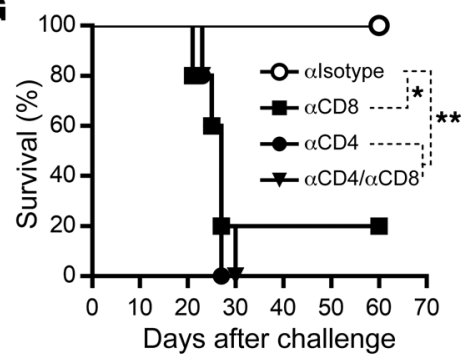

Figure 5. Both endogenous $\mathrm{CD4}^{+}$and $\mathrm{CD8}^{+} \mathrm{T}$ cells are required to prevent tumor escape during combination therapy. CMS5 TB BALB/C mice were depleted of specific lymphocyte populations via treatment with the indicated antibody or CPX concurrent with treatment with combination therapy, and resulting tumor growth (A) and survival (B) were monitored. Antibodies were given 1 day before and 1 day after T cell transfer and once a week thereafter for 3 weeks. A single injection of CPX was given 1 day before T cell transfer. (C) Frequency of antigen-spreading CD8 ${ }^{+} \mathrm{T}$ cell responses in the peripheral blood were quantified via stimulation with a pool of 4 peptides corresponding to previously identified CMS5 neoepitopes (27) and staining for IFN- $\gamma$ production. (D) Frequency of ErkM-specific T cells in the peripheral blood of BALB/c mice surviving initial CMS5 tumor challenge after combination therapy (60+ days) was assessed before and 5 days after rechallenge with CMS5 or CM5 relapse (CMS5r) cells, and resultant survival (E) is also shown. Naive mice receiving the CMS5 or CMS5r challenge were included as controls. (F) Survival of mice vaccinated with lethally irradiated CMS5r cells (irrCMS5r) before challenge with CMS5 or CMS5r cells. (G) Survival of tumor-regressed mice (as described above) rechallenged with CMS5r after depletion of lymphocyte populations with the indicated antibodies. Data are shown as representative of 2 independent experiments (A-E and $\mathbf{G})$ or a single experiment $(\mathbf{F})$ with $n=5$ per group. Data were analyzed using 1-way ANOVA with Holm-Šidák correction for multiple comparisons (C), paired 2-tailed $t$ test (D), and log-rank (Mantel-Cox) test (B and $\mathbf{E}-\mathbf{G}) .{ }^{*} P<0.05 ;{ }^{* *} P<0.01 ;{ }^{* *} P<0.001$.

backbone to encode a surrogate neoantigen gp33, the immunodominant peptide from the lymphocytic choriomeningitis virus glycoprotein, and a murine B16 melanoma cell line engineered to express gp33 (B16-gp33). C57BL/6 mice bearing i.d. B16gp33 tumors were treated with Tcm derived from gp33-specific P14 TCR transgenic T cells followed by VacV-gp33 vaccination. As shown in Figure 6A, complete and durable tumor regression was achieved in mice that received combination therapy, confirming the potency and flexibility of this combination platform for targeting different tumor antigens and/or incorporating different OV backbones. Interestingly, however, mice treated with either anti-Thy1.2 antibody or CPX prior to combination therapy showed significantly reduced survival due to tumor relapse after initial regression (Figure 6, A and B), reinforcing the importance of preexisting host $\mathrm{T}$ lymphocytes that likely broaden diversity of the immune attack.

\section{Discussion}

Strategies to selectively enhance $\mathrm{T}$ cell reactivity against genetically defined neoantigens are currently under development (29, 30). In the present study, we explored a therapeutic platform combining ex vivo-expanded neoantigen-specific T cells (ACT) with an OVV. This strategy employs a "push-pull" mechanism in which the OVV facilitates both $\mathrm{T}$ cell activation and expansion in the periphery (push), followed by the recruitment of $\mathrm{T}$ cells to the tumor site (pull). Indeed, we demonstrated that adoptive transfer of antigen-specific Tcm followed by an oncolytic vaccine elicited robust $\mathrm{T}$ cell expansion, tumor infiltration, and complete tumor regression, revealing a powerful synergy between these 2 therapeutic approaches. More importantly, the efficacy was achieved in the absence of preconditioning (i.e., total body irradiation or lymphodepleting chemotherapy prior to the cell transfer) and exogenous IL-2, 2 adjuvants that are typically employed in other ACT 

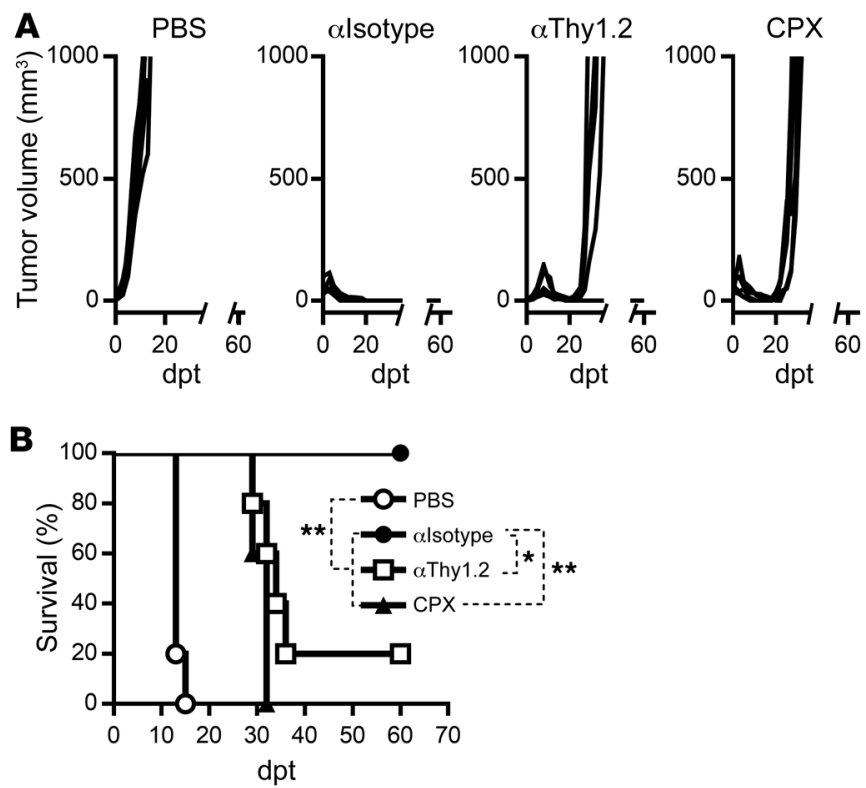

Figure 6. Requirement of endogenous T lymphocytes for preventing tumor relapse is evidenced in a different model. C57BL/ 6 mice bearing 6-day-old i.d. B16-gp33 tumors were given ACT ( $10^{6} \mathrm{gp} 33$-specific Tcm) followed by vaccination with $5 \times 10^{7}$ PFU VacV-gp33 (both given i.v.). Antibodies were given 1 day before and 1 day after $T$ cell transfer and once a week thereafter for 3 weeks. A single injection of CPX was given 1 day before ACT. (A) Tumor volumes and (B) survival were monitored and are shown. Data are shown as representative results of 2 independent experiments with $n=5$ per group. Data were analyzed using a log-rank (Mantel-Cox) test $(\mathbf{B}) .{ }^{*} P<0.05 ;{ }^{*} P<0.01$

settings $(15,16)$, highlighting the translational implication of our combination therapy that may offer a less intensive experience for the patient. Furthermore, and most importantly, bypassing preconditioning preserves tumor-primed endogenous $\mathrm{T}$ cells that not only complement ACT to eliminate the primary tumor and prevent the emergence of antigen-loss variants, but also form a longterm memory pool for immune surveillance.

Despite the success in treating B cell malignancies and melanoma, ACT has only limited effects on most solid tumors $(31,32)$. Hurdles include, among other aspects, inefficiency of transferred $\mathrm{T}$ cells in infiltrating the tumor in sufficient quantity and persisting long enough to kill all malignant cells $(33,34)$. Increasing the dose of transferred cells may improve their ability to access and kill solid tumors, but generating a large number of $\mathrm{T}$ cells ex vivo requires extensive expansion that inevitably results in terminal differentiation and replicative senescence of $\mathrm{T}$ cells $(35,36)$. Furthermore, the highly suppressive microenvironment and heterogeneous antigen landscape associated with solid tumors often render T cells ineffectual and promote antigen escape $(37,38)$. Thus, to achieve sustained regression of solid tumors, ACT must be combined with other approaches that can simultaneously stimulate $\mathrm{T}$ cell expansion, recruit $\mathrm{T}$ cells into the tumor, overcome tumor-mediated immunosuppression, and broaden spectrum of $\mathrm{T}$ cell specificity. We have previously demonstrated that oncolytic vaccines can effectively expand tumor-specific Tcm while retaining their beneficial oncolytic properties, which led us to hypothesize that OVVs might represent an ideal platform for combining with ACT due to their vaccination and oncolytic functions $(18,19)$. Indeed, we provide evidence in this study that neither OV (no tumor antigen) nor conventional vaccine (nononcolytic) was sufficient to synergize with $\mathrm{ACT}$ and that destruction of established solid tumors required both amplification and tumor infiltration of transferred $\mathrm{T}$ cells. To this end, Tcm were superior to Teff due to their efficient engraftment and proliferative capacity, consistent with an increasingly appreciated concept in the field of ACT $(35,36)$.

We made 2 additional observations that may have important implications in the current clinical practice and rational design of ACT. First, although tumor regression was achieved in immunodeficient mice following ACT plus OV vaccination, tumors reoccurred within 2 weeks. It was evident that the relapsed tumor cells no longer harbored the epitope target of the transferred $\mathrm{T}$ cells. This outcome seems to support the notion that targeting tumors with a $T$ cell population specific for a restricted set of antigens may lead to the selective outgrowth of antigen-negative tumor variants. However, durable regression was consistently achieved in WT animals, suggesting that tumor heterogeneity and/or immune escape can be addressed by mobilizing endogenous $\mathrm{T}$ cells during ACT, even targeting a single antigen. Our observation that depletion of $\mathrm{CD} 4^{+}$or $\mathrm{CD} 8^{+} \mathrm{T}$ cells prior to ACT plus OV treatment did not affect initial tumor regression, but resulted in relapse, suggests that there is cooperation between transferred $\mathrm{T}$ cells and endogenous $\mathrm{T}$ cells. It appears that transferred $\mathrm{T}$ cells play a primary role in mediating tumor debulking (eradication of $\mathrm{ErkM}^{+}$tumor cells), while endogenous $\mathrm{T}$ cells are required to eliminate escaped variants (ErkM- tumor cells). One possible mechanism that explains activation of endogenous $\mathrm{T}$ cells is epitope spreading, a phenomenon involving in vivo crosspresentation of tumor-derived antigens released in one wave of immune attack to promote subsequent rounds of antitumor $\mathrm{T}$ cells against different antigens $(14,39)$. These sequential events may be particularly effective in our case due to robust tumor lysis and inflammation mediated by transferred $\mathrm{T}$ cells and OVs. However, another plausible mechanism is that preexisting tumor-primed $\mathrm{T}$ cells are unleashed and/or expanded by ACT plus OV therapy, thus providing a broader repertoire to complement transferred $\mathrm{T}$ cells for complete eradication of all tumor cells. This latter possibility is supported by several lines of evidence. First, the presence and boosting of ErkM-specific $\mathrm{CD} 8^{+} \mathrm{T}$ cells could be detected as early as 2 days after ACT plus OVV. Second, the increased $\mathrm{T}$ cell responses to nontargeted neoepitopes were also manifested 5 days after combination therapy. These observations are unlikely to be the result of epitope spreading that requires activation of naive $\mathrm{T}$ cells. Furthermore, the fact that depletion of $\mathrm{CD} 4^{+}$or $\mathrm{CD} 8^{+} \mathrm{T}$ cells 6 days after treatment did not result in relapse suggests that preexisting, but not subsequently induced, host $\mathrm{T}$ cells are involved in early clearance of tumor cells in cooperation with transferred T cells. Nevertheless, it is likely that ACT plus OV can both reinvigorate preexisting antigen-specific $\mathrm{T}$ cells and induce new $\mathrm{T}$ cell responses via epitope spreading in a sequential manner that reflects their relative importance during the generation of antitumor immunity (39, 40). More work is needed in both scenarios to determine whether those unidentified antigens recognized by naturally developing or therapeutically induced $\mathrm{T}$ cells are derived from tumor-specific mutations or self-antigens. 
Second, long-term persistence of transferred T cells is considered important, and it has been reported that there is a positive correlation between tumor regression and the degree of persistence of adoptively transferred $\mathrm{T}$ cell clones $(41,42)$. Interestingly, however, our transferred anti-ErkM CD8 ${ }^{+} \mathrm{T}$ cells disappeared right after tumor regression, while endogenous ErkM-specific CD8 ${ }^{+}$ $\mathrm{T}$ cells survived long term and were capable of providing antigen-specific protective immunity. We speculate that the observation in our study is due to the introduction of oncolytic vaccination that not only accelerates transferred $\mathrm{T}$ cell responses and intensifies their interactions with tumors, but also engages endogenous antitumor $\mathrm{T}$ cell responses. Indeed, we demonstrated that transferred $\mathrm{T}$ cells dominated early expansion and tumor infiltration and were responsible for mediating initial tumor regression. As a consequence, transferred T cells underwent tumor-induced apoptosis, a phenomenon documented by previous studies $(43,44)$. In contrast, endogenous $\mathrm{T}$ cell expansion did not reach its peak until several days after tumor regression, suggesting that the majority of boosted and/or therapeutically induced endogenous T cells did not experience interactions with tumor cells and thereby survived to maintain antitumor immunity. The fact that transferred $\mathrm{T}$ cells in $\mathrm{TF}$ mice survived long term confirms that the short-lived fate of transferred T cells in TB mice is likely the result of their interaction with tumor cells. Our results argue that preserving endogenous tumor-reactive $\mathrm{T}$ cells during ACT is vital to ensuring disease elimination and long-term antitumor memory, which may be compromised by preconditioning in an attempt to augment the survival of adoptively transferred cells (16).

Collectively, our data support a possibility of harnessing the $\mathrm{T}$ cell repertoire by engaging tumor-primed host $\mathrm{T}$ lymphocytes during ACT and thus minimizing or even eliminating the risk of antigen-negative outgrowth that results from the introduction of a singular selective pressure. The fact that many patients have $\mathrm{CD}^{+}$and/or $\mathrm{CD}^{+} \mathrm{T}$ cells that recognize different neoepitopes derived from patients' own tumors underscores the relevance of our findings $(45,46)$. Although it remains to be seen how well our results can be translated from mouse models into the clinic, there is mounting evidence for the relevance of OV-induced antigen spreading and the development of endogenous $\mathrm{T}$ cell responses in parallel with ACT in the clinic $(47,48)$. Moreover, clinical methodologies to generate tumor-specific Tcm from cancer patients have been developed, together with established safety profiles of various OVs, supporting the idea that our combination therapy is highly clinically translatable $(7-9,49,50)$.

\section{Methods}

Animals. BALB/c or C57BL/6 mice were purchased from Charles River Laboratories and housed in a specific pathogen-free room in the McMaster University Central Animal Facility. NRG (NOD.Cg-Rag1 $1^{\text {tm1Mom }}$ $I l 2 \mathrm{rg}^{\mathrm{tm} 1 \mathrm{Wi}} / \mathrm{SzJ}$ ) breeders were purchased from the Jackson Laboratory, and mice were bred under ultraclean conditions. DUC18 mice were provided by Lyse Norian (University of Iowa, Iowa City, Iowa, USA) (22). B6.Cg-Tcratm1Mom Tg (TcrLCMV)327Sdz (P14) mice were purchased from Taconic Breeding Laboratories.

Viral vectors. Recombinant VSV was engineered to express an $\mathrm{H}-2 \mathrm{~K}^{\mathrm{d}}$-restricted epitope corresponding to amino acids $136-144$ of a mutated ERK2 protein $\left(\operatorname{ErkM}_{136-144}\right)$, and the resultant VSV vaccine vector was dubbed VSV-ErkM. VSV-MT is a control vector that lacks a transgene. Ad-ErkM is a replication-deficient, E1/E3-deleted adenoviral vector containing the ErkM epitope. VacV-gp33 is a TK-deleted recombinant VacV (Western Reserve strain) expressing gp33, an $\mathrm{H}-2 \mathrm{D}^{\mathrm{b}}$-restricted epitope derived from the lymphocytic choreomeningitis virus glycoprotein (51).

Peptides. Peptides for ErkM (QYIHSANVL), gp33 (KAVYNFATM), Alkbh6.2 (DVPMEQPR), Slit3 (GFHGCIHEVI), Atxn10.1 (QVFPGLMEI), and Ccdc136 (ELQGLLEDEI) were purchased from Biomer Technologies and dissolved in PBS supplemented with 0.5\% BSA.

Cell lines and tumor challenge. All cells were maintained at $37^{\circ} \mathrm{C}$ in a humidified atmosphere with $5 \% \mathrm{CO}_{2}$. CMS5 (a gift from Lyse Norian) (22), CMS5r, and CMS5r-LVErkM cells were cultured in DMEM supplemented with $10 \% \mathrm{FBS}$, penicillin/streptomycin $(100 \mathrm{U} / \mathrm{mL}$ and 100 $\mathrm{ng} / \mathrm{mL}$, respectively), and $2 \mathrm{mM}$ L-glutamine (Thermo Fisher Scientific). B16-gp33 cells (B16F10 cells stably transfected with a minigene corresponding to the gp33 peptide) (52) were maintained in MEM/F11 containing 10\% FBS, $2 \mathrm{mM}$ L-glutamine, $5 \mathrm{ml}$ sodium pyruvate, $5 \mathrm{~mL}$ nonessential amino acids, $5 \mathrm{~mL}$ vitamin solution (Thermo Fisher Scientific), $55 \mu \mathrm{M}$ 2-mercaptoethanol (Sigma-Aldrich), $100 \mathrm{U} / \mathrm{mL}$ penicillin, and $100 \mathrm{ng} / \mathrm{ml}$ streptomycin.

Tumor cells were washed twice with PBS and resuspended in PBS at a concentration of $10^{6}$ cells $/ 30 \mu \mathrm{L}$ for CMS5 cells or $10^{5}$ cells $/ 30 \mu \mathrm{L}$ for B16-gp33 cells. Mice were challenged via i.d. injection, and tumors were allowed to grow to a mean volume of approximately $150 \mathrm{~mm}^{3}$ prior to the commencement of treatment. Relapsed CMS5 cells (termed CMS5r) were obtained from end-point NRG mice. CMS5r-LVErkM were generated via transduction of CMS5r with a lentivirus engineered to expresses the ErkM peptide by annealing $5^{\prime}$ phosphorylated primers (ErkM, forward, GATCCATGCAATACATACACTCAGCTAACGTGTTGTAAG; ErkM, reverse, AATTCTTACAACACGTTAGCTGAGTGTATGTATTGCATG) before ligation into the BamHI and EcoRI sites of pLV-EF1a-IRES-Puro (plasmid 8513 from Addgene; ref. 53). After transduction, a polyclonal cell line was generated using puromycin selection (Invitrogen).

For lethal irradiation vaccination experiments, CMS5r cells were trypsinized, suspended in PBS at $3 \times 10^{7}$ cells $/ \mathrm{mL}$, and exposed to 150 Gy using a Gammacell 1000 (Best Theratronics Ltd.) with a Cs-137 source before i.d. injection of approximately $10^{6}$ cells per mouse. Mice were vaccinated twice at one-week intervals and challenged with $2 \times$ $10^{5}$ live cells 1 week after the second vaccination.

PCR analysis. Genomic DNA was extracted from CMS5, CMS5r, and CT26 cells using Purelink Genomic DNA Extraction Kits (Life Technologies) according to the manufacturer's instructions. PCR was carried out on extracted DNA using ERK2 primers (ERK2, forward, 5'-TGTGCCGTGTTCTCTTCAGT-3', ERK2, reverse, 5'-TGACTTGGCTGACCTTGAGA-3') with the Q5 High-Fidelity DNA Polymerase Kit (New England Biolabs) per the manufacturer's instructions, on a T3000 Thermocycler (Biometra). The amplification program was as follows: $98^{\circ} \mathrm{C}$ for 30 seconds, then $98^{\circ} \mathrm{C}$ for 10 seconds, $58^{\circ} \mathrm{C}$ for 30 seconds, $72^{\circ} \mathrm{C}$ for 30 seconds ( $\times 35$ cycles), with final elongation at $72^{\circ} \mathrm{C}$ for 2 minutes. The amplification products were digested with Sfc1 and run on a 1\% UltraPure agarose gel (Life Technologies) with EZ-Vision loading buffer/dye (Amresco). The PCR product from each cell line was sequenced using the forward primer described above.

In vitro $T$ cell differentiation. Bulk splenocytes from transgenic mice were isolated and cultured for 7 days in the presence of 100 
$\mathrm{ng} / \mathrm{mL}$ of ErkM or gp33 peptide. For Tcm differentiation, $10 \mathrm{ng} / \mathrm{mL}$ IL-15, $10 \mathrm{ng} / \mathrm{mL}$ IL-21 (BioLegend), and $20 \mathrm{ng} / \mathrm{mL}$ rapamycin (SigmaAldrich) were added to the culture, while 60 units/mL IL-2 (BioLegend) was used to produce Teff.

Combination therapy. When tumors reached an approximate mean volume of $150 \mathrm{~mm}^{3}$, in vitro-differentiated $\mathrm{CD} 8^{+}$transgenic $\mathrm{T}$ cells were injected i.v. into TB mice at a dose of $10^{6}$ cells $/ 200 \mu \mathrm{L}$ of PBS. After 24 hours, mice were treated with different vaccines. VSV (2 $\left.\times 10^{8} \mathrm{PFU}\right)$ and vaccinia $\left(7 \times 10^{7} \mathrm{PFU}\right)$ vectors were delivered i.v. and Ad-ErkM $\left(5 \times 10^{8} \mathrm{PFU}\right)$ was injected intramuscularly. Peptide vaccination consisted of $150 \mu \mathrm{g}$ synthetic Erk peptide, $100 \mu \mathrm{g}$ anti-CD40 $\mathrm{Ab}$ (clone FGK4.5/FGK45, Bio X Cell), and $50 \mu \mathrm{g}$ poly-IC (SigmaAldrich), administered as a mixture via the i.v. route as previously optimized by Cho et al. (25).

Surface and intracellular staining of $T$ cells. The following stains and Abs for flow cytometric analysis were purchased from BD Biosciences: Fc block (catalog 553141), 7AAD (catalog 559925), Fixable Viability Stain 510 (catalog 564406), Pacific Blue rat anti-mouse CD8a (clone 558106), APC rat anti-mouse IFN- $\gamma$ (clone XMG1.2), PE rat anti-mouse CD4 (clone GK1.5), PE mouse anti-rat Thy1.1 (clone OX-7), Alexa Fluor 700 rat anti-mouse CD62L (clone MEL-14), and FITC rat anti-mouse CD44 (clone IM-7). Blood, spleen, and bone marrow samples were collected and treated with ACK lysis buffer to remove red blood cells prior to peptide stimulation and/or staining. Cells were treated with Fc Block and stained for surface markers followed by viability staining. For analysis of antigen-specific responses, PBMCs were extracted from blood samples using RBC lysis buffer and stimulated with ErkM or gp33 peptide $(1 \mu \mathrm{g} / \mathrm{mL})$ in culture at $37^{\circ} \mathrm{C}$ for 4 hours. Brefeldin A (GolgiPlug, BD Biosciences; $1 \mu \mathrm{g} / \mathrm{mL}$ ) was added for the last 3 hours of incubation. Blocking and surface staining were performed as above except that the cells were stained with fixable viability dye before fixation/permeabilization (Cytofix/Cytoperm, BD Biosciences) and intracellular staining fluorescence was detected using either a BD LSRFortessa or LSR II flow cytometer (BD Biosciences). Data were analyzed using FlowJo (version 10) flow cytometry analysis software (Tree Star).

Cytotoxicity assay. CMS5r-LVErlM cells were labeled with $5 \mu \mathrm{M}$ CFSE (MilliporeSigma, catalog 21888), seeded in a 96-well plate at $10^{5}$ cells per well, and cocultured with DUC18 Teffs (generated as above) at the indicated ratios for 16 hours. Cells were stained with eBioscience Fixable Viability Dye eFluor 780 (catalog 65-0865-14, Thermo Fisher), and staining was evaluated by flow cytometry as above. The percentage of specific lysis was then calculated using the following equation: $\%$ specific lysis $=100 \times(\%$ specific cell death $-\%$ basal cell death) $/ 100$ - \%basal cell death, where specific cell death is determined from viability dye staining of CFSE-positive cells in T cell coculture wells and basal cell death from wells lacking cocultured $\mathrm{T}$ cells

In vivo depletion of $T$ lymphocytes. Depletion of $\mathrm{T}$ cells was achieved by 2 i.p. injections of anti-mouse CD4 and CD8 (produced from GK1.5 and 2.43 hybridomas, respectively, purchased from ATCC) or Thy1.2 antibody (clone $30 \mathrm{H} 12$, Bio X Cell) at a dose of $250 \mu \mathrm{g} / 200 \mu \mathrm{L}$ spaced 48 hours apart. Treatment with isotype control antibody (clone HRPN,
Bio X Cell) followed the same pattern. For longer observations, depletion was maintained using a biweekly i.p. injection of corresponding antibodies. CPX (Sigma-Aldrich) was i.p. administered 1 day prior to $\mathrm{T}$ cell transfer at a dose of $3 \mathrm{mg} / \mathrm{mouse}$. Depletion efficiency was monitored by FACS analysis of PBMCs at different time points during treatment (data not shown).

Histology. Immunohistochemistry was performed on sections from formalin-fixed, paraffin-embedded tumor tissues using a Leica Bond Rx automated stainer (Leica Biosystem). Slides were dewaxed and pretreated with Leica Bond Epitope Retrieval buffer \#2 (Leica Biosystems) for 20 minutes before staining with rat anti-mouse CD8 $\alpha$ antibody (diluted 1:1000; clone 4SM15, Thermo Fisher Scientific). Color was developed using the Leica Bond Polymer Refine Detection Kit (Leica Biosystems), substituting the postprimary component with rabbit antirat antibody (1:100, Vector Laboratories). Images where taken using an Axiovert 100M microscope (Zeiss), and quantification was performed using the analyze particles function of ImageJ software (54).

Statistics. GraphPad Prism for Windows was used for graphing and statistical analyses. Differences between means of immune response data were queried using either paired Student's 2-tailed $t$ test, 1-way ANOVA, or repeated measures 2-way ANOVA as described in the figure legends. Where necessary, the Holm-Šidák method was used to correct for multiple comparisons. Overall, a $P$ value of less than 0.05 was considered significant. Mean + SD bars are shown. Survival curves were generated using the Kaplan-Meier method, with a tumor volume of $1000 \mathrm{~mm}^{2}$ or tumor ulceration as end point, and analyzed using the log-rank (Mantel-Cox) test.

Study approval. All animal experiments were compliant with Canadian Council on Animal Care guidelines and received internal approval through the McMaster University Animal Research Ethics Board.

\section{Author contributions}

SRW, BS, LC, DB, AN, and TSM performed the experiments, analyzed the data, and assisted in manuscript preparation. KS and BDL constructed the viral vectors and assisted in data analysis. JLB, SRW, and YW oversaw experimental design and data interpretation and prepared the manuscript.

\section{Acknowledgments}

We thank Lyse Norian for providing DUC18 mice. This study was conducted with the support of the Ontario Institute for Cancer Research through funding provided by the Government of Ontario, as well as the Canadian Institutes of Health Research (FRN 123516 and FRN 152954), the Canadian Cancer Society (grant 705143), and the Terry Fox Research Institute (TFRI-1073).

Address correspondence to: Yonghong Wan, Department of Pathology and Molecular Medicine, McMaster University, Room MDCL-5024, 1200 Main Street West, Hamilton, Ontario, Canada, L8N 3Z5. Phone: 905.525.9140 ext.22461; Email: wanyong@ momaster.ca.
1. Schumacher TN, Schreiber RD. Neoantigens in cancer immunotherapy. Science. 2015;348(6230):69-74.

2. Chabanon RM, Pedrero M, Lefebvre C, Marabelle A, Soria JC, Postel-Vinay S. Mutational landscape and sensitivity to immune checkpoint blockers. Clin Cancer Res. 2016;22(17):4309-4321.

3. Snyder A, et al. Genetic basis for clinical response to CTLA-4 blockade in melanoma. N Engl J Med. 2014;371(23):2189-2199.
4. Hugo W, et al. Genomic and transcriptomic features of response to anti-PD-1 therapy in metastatic melanoma. Cell. 2016;165(1):35-44.

5. McGranahan N, et al. Clonal neoantigens elicit $\mathrm{T}$ cell immunoreactivity and sensitiv- 
ity to immune checkpoint blockade. Science. 2016;351(6280):1463-1469.

6. Houot R, Schultz LM, Marabelle A, Kohrt H. T-cell-based immunotherapy: adoptive cell transfer and checkpoint inhibition. Cancer Immunol Res. 2015;3(10):1115-1122.

7. Pollack SM, et al. Tetramer guided, cell sorter assisted production of clinical grade autologous NY-ESO-1 specific CD8(+) T cells. J Immunother Cancer. 2014;2(1):36.

8. Kaiser AD, et al. Towards a commercial process for the manufacture of genetically modified T cells for therapy. Cancer Gene Ther. 2015;22(2):72-78.

9. Sadelain M, Rivière I, Riddell S. Therapeutic T cell engineering. Nature. 2017;545(7655):423-431.

10. Maude SL, et al. Chimeric antigen receptor $T$ cells for sustained remissions in leukemia. $N$ Engl JMed. 2014;371(16):1507-1517.

11. Robbins PF, et al. A pilot trial using lymphocytes genetically engineered with an NY-ESO-1reactive T-cell receptor: long-term follow-up and correlates with response. Clin Cancer Res. 2015;21(5):1019-1027.

12. Louis CU, et al. Antitumor activity and longterm fate of chimeric antigen receptor-positive $\mathrm{T}$ cells in patients with neuroblastoma. Blood. 2011;118(23):6050-6056.

13. Bollard CM, et al. Sustained complete responses in patients with lymphoma receiving autologous cytotoxic T lymphocytes targeting Epstein-Barr virus latent membrane proteins. JClin Oncol. 2014;32(8):798-808.

14. Chapuis AG, et al. T-cell therapy using interleukin-21-primed cytotoxic T-cell lymphocytes combined with cytotoxic T-cell lymphocyte antigen-4 blockade results in long-term cell persistence and durable tumor regression. JClin Oncol. 2016;34(31):3787-3795.

15. Ledford H. Safety concerns blight promising cancer therapy. Nature. 2016;538(7624):150-151.

16. Muranski P, et al. Increased intensity lymphodepletion and adoptive immunotherapy--how far can we go? Nat Clin Pract Oncol. 2006;3(12):668-681.

17. Wrzesinski C, et al. Increased intensity lymphodepletion enhances tumor treatment efficacy of adoptively transferred tumor-specific $\mathrm{T}$ cells. J Immunother. 2010;33(1):1-7.

18. Bridle BW, et al. Potentiating cancer immunotherapy using an oncolytic virus. Mol Ther. 2010;18(8):1430-1439.

19. Bridle BW, et al. Privileged antigen presentation in splenic $b$ cell follicles maximizes $\mathrm{T}$ cell responses in prime-boost vaccination. J Immunol. 2016;196(11):4587-4595.

20. Walsh SR, et al. Type I IFN blockade uncouples immunotherapy-induced antitumor immunity and autoimmune toxicity. JClin Invest. 2019;129(2):518-530.

21. Pol JG, et al. Maraba virus as a potent oncolytic vaccine vector. Mol Ther. 2014;22(2):420-429.

22. Ikeda $\mathrm{H}$, et al. Mutated mitogen-activated protein kinase: a tumor rejection antigen of mouse sarcoma. Proc Natl Acad Sci USA. 1997;94(12):6375-6379.

23. Hanson HL, et al. Eradication of established tumors by $\mathrm{CD} 8+\mathrm{T}$ cell adoptive immunotherapy. Immunity. 2000;13(2):265-276.

24. Klebanoff CA, et al. Central memory self/ tumor-reactive CD8+ T cells confer superior antitumor immunity compared with effector memory T cells. Proc Natl Acad Sci USA. 2005;102(27):9571-9576.

25. Cho HI, Jung SH, Sohn HJ, Celis E, Kim TG. An optimized peptide vaccine strategy capable of inducing multivalent $\mathrm{CD} 8^{+} \mathrm{T}$ cell responses with potent antitumor effects. Oncoimmunology. 2015;4(11):e1043504.

26. North RJ. Cyclophosphamide-facilitated adoptive immunotherapy of an established tumor depends on elimination of tumor-induced suppressor $\mathrm{T}$ cells. JExp Med. 1982;155(4):1063-1074.

27. Salem ML, et al. Defining the ability of cyclophosphamide preconditioning to enhance the antigen-specific CD8+ T-cell response to peptide vaccination: creation of a beneficial host microenvironment involving type I IFNs and myeloid cells. J Immunother. 2007;30(1):40-53.

28. Duan F, et al. Genomic and bioinformatic profiling of mutational neoepitopes reveals new rules to predict anticancer immunogenicity. JExp Med. 2014;211(11):2231-2248.

29. Tran E, Robbins PF, Rosenberg SA. 'Final common pathway' of human cancer immunotherapy: targeting random somatic mutations. Nat Immunol. 2017;18(3):255-262.

30. Yarchoan M, Johnson BA, Lutz ER, Laheru DA, Jaffee EM. Targeting neoantigens to augment antitumour immunity. Nat Rev Cancer. 2017;17(4):209-222.

31. Fousek K, Ahmed N. The evolution of T-cell therapies for solid malignancies. Clin Cancer Res. 2015;21(15):3384-3392.

32. Gilham DE, Debets R, Pule M, Hawkins RE, Abken H. CAR-T cells and solid tumors: tuning $\mathrm{T}$ cells to challenge an inveterate foe. Trends $\mathrm{Mol}$ Med. 2012;18(7):377-384.

33. Restifo NP, Dudley ME, Rosenberg SA. Adoptive immunotherapy for cancer: harnessing the $\mathrm{T}$ cell response. Nat Rev Immunol. 2012;12(4):269-281.

34. Wu R, et al. Adoptive T-cell therapy using autologous tumor-infiltrating lymphocytes for metastatic melanoma: current status and future outlook. Cancer J. 2012;18(2):160-175.

35. Busch DH, Fräßle SP, Sommermeyer D, Buchholz VR, Riddell SR. Role of memory T cell subsets for adoptive immunotherapy. Semin Immunol. 2016;28(1):28-34.

36. Crompton JG, Sukumar M, Restifo NP. Uncoupling T-cell expansion from effector differentiation in cell-based immunotherapy. Immunol Rev. 2014;257(1):264-276.

37. Junttila MR, de Sauvage FJ. Influence of tumour micro-environment heterogeneity on therapeutic response. Nature. 2013;501(7467):346-354.

38. Gajewski TF, Schreiber H, Fu YX. Innate and adaptive immune cells in the tumor microenvironment. Nat Immunol. 2013;14(10):1014-1022.

39. Corbière V, et al. Antigen spreading contributes to MAGE vaccination-induced regression of melanoma metastases. Cancer Res. 2011;71(4):1253-1262.

40. Boon T, Coulie PG, Van den Eynde BJ, van der Bruggen P. Human T cell responses against melanoma. Аnпи Rev Immunol. 2006;24:175-208.

41. Chapuis AG, et al. Tracking the fate and origin of clinically relevant adoptively transferred $\mathrm{CD} 8^{+} \mathrm{T}$ cells in vivo. Sci Immunol. 2017;2(8):eaal2568.

42. Robbins PF, et al. Cutting edge: persistence of transferred lymphocyte clonotypes correlates with cancer regression in patients receiving cell transfer therapy. JImmunol. 2004;173(12):7125-7130.

43. Norian LA, Allen PM. Rapid maturation of effector $\mathrm{T}$ cells in tumors, but not lymphoid organs, during tumor regression. PLoS One. 2007;2(9):e821.

44. Radoja S, Saio M, Frey AB. CD8+ tumor-infiltrating lymphocytes are primed for Fas-mediated activation-induced cell death but are not apoptotic in situ. J Immunol. 2001;166(10):6074-6083.

45. Tran E, et al. Cancer immunotherapy based on mutation-specific CD4+ T cells in a patient with epithelial cancer. Science. 2014;344(6184):641-645.

46. Tran E, et al. T-cell transfer therapy targeting mutant KRAS in cancer. $N$ Engl J Med. 2016;375(23):2255-2262.

47. Kaufman HL, Kim DW, DeRaffele G, Mitcham J, Coffin RS, Kim-Schulze S. Local and distant immunity induced by intralesional vaccination with an oncolytic herpes virus encoding GM-CSF in patients with stage IIIc and IV melanoma. Ann Surg Oncol. 2010;17(3):718-730.

48. Ma L, et al. Enhanced CAR-T cell activity against solid tumors by vaccine boosting through the chimeric receptor. Science. 2019;365(6449):162-168.

49. Breitbach CJ, et al. Intravenous delivery of a multi-mechanistic cancer-targeted oncolytic poxvirus in humans. Nature. 2011;477(7362):99-102.

50. LeBlanc AK, et al. Safety studies on intravenous administration of oncolytic recombinant vesicular stomatitis virus in purpose-bred beagle dogs. Hum Gene Ther Clin Dev. 2013;24(4):174-181.

51. Oldstone MB, Tishon A, Eddleston M, de la Torre JC, McKee T, Whitton JL. Vaccination to prevent persistent viral infection. J Virol. 1993;67(7):4372-4378.

52. Prévost-Blondel A, Zimmermann C, Stemmer C, Kulmburg P, Rosenthal FM, Pircher H. Tumor-infiltrating lymphocytes exhibiting high ex vivo cytolytic activity fail to prevent murine melanoma tumor growth in vivo. J Immunol. 1998;161(5):2187-2194.

53. Hayer A, et al. Engulfed cadherin fingers are polarized junctional structures between collectively migrating endothelial cells. Nat Cell Biol. 2016;18(12):1311-1323.

54. Schindelin J, et al. Fiji: an open-source platform for biological-image analysis. Nat Methods. 2012;9(7):676-682. 